\title{
CISTEÍNOPROTEASAS CATEPSINAS L DE Taenia solium: ROL BIOLÓGICO EN LA INFECCIÓN Y POTENCIAL USO PARA EL INMUNODIAGNÓSTICO DE LA NEUROCISTICERCOSIS
}

\author{
Nancy León ${ }^{1, a}$, Carlos Padilla ${ }^{2, a}$, Mónica Pajuelo ${ }^{1, b}$, Patricia Sheen ${ }^{1, a, b}$, \\ Mirko Zimic ${ }^{1, b, c}$
}

RESUMEN

Taenia solium es un helminto aplanado responsable de la teniosis y de la cisticercosis humana, siendo esta última producida por el consumo de huevos infectivos. Los cisticercos pueden desarrollarse en diferentes tejidos del hombre, frecuentemente en el sistema nervioso central causando la neurocisticercosis (NCC). Para el diagnóstico de la NCC se requiere de una adecuada interpretación de datos clínicos, resultados de neuroimagen y pruebas serológicas. Sin embargo, las pruebas serológicas podrían mejorarse con el desarrollo de antígenos candidatos capaces de incrementar su sensibilidad y especificidad. En los últimos años se han descrito una serie de proteínas de superficie y de secreción de $T$. solium esenciales para la interacción parásito-hospedero. Una de estas familias son las cisteínoproteasas catepsinas L, las cuales cumplen un rol preponderante para el desarrollo y supervivencia del parásito, participando en la invasión tisular, la evasión de la respuesta inmune, el desenquistamiento y enquistamiento del cisticerco. Son consideradas como antígenos potenciales para el inmunodiagnóstico de la neurocisticercosis.

Palabras clave: Catepsina L; Taenia solium; Interacciones huésped-parásitos; Pruebas inmunológicas; Neurocisticercosis (fuente: DeCS BIREME)

\section{CATHEPSIN L CYSTEINE PROTEASE FROM Taenia solium: ITS BIOLOGICAL ROLE IN THE INFECTION AND POTENTIAL USE FOR THE IMMUNODIAGNOSIS OF NEUROCYSTICERCOSIS}

\section{ABSTRACT}

Taenia solium is a plane helminth responsible for taeniasis and human cysticercosis, the latter being the result of the consumption of infective eggs. Cysticerci can develop in different human tissues, often in the central nervous system, causing neurocysticercosis (NCC). For the diagnosis of NCC, an adequate interpretation of clinical data, neuroimaging results and serological tests are required. However, serological tests could be improved by developing candidate antigens able to increase their sensibility and specificity. In the last years, a series of surface and secretory proteins of $T$. solium essential for the parasite-host interaction have been described. One of these families is cathepsin $L$ cysteine proteases, which have a predominant role in the development and survival of the parasite. They take part in the tissue invasion, immune response evasion, excystation and encystment of cysticercus. They are considered potential antigens for the immunodiagnosis of neurocysticercosis.

Key words: Cathepsin L; Taenia solium; Host-parasite interactions; Immunologic tests; Neurocysticercosis (source: MeSH NLM).

\section{INTRODUCCIÓN}

Taenia solium pertenece a la familia Taeniidae, de la clase Céstoda, del filum Platyhelminthes, cuyo estadio adulto causa la teniosis, y su estadio larvario (cisticerco o metacéstodo) causa la cisticercosis ${ }^{(1)}$. La teniosis no produce sintomatología grave; por el contrario, la cisticercosis del sistema nervioso central, denominada neurocisticercosis (NCC), se presenta con sintomatología diversa, pudiendo cursar con convulsiones, mareos, dolor de cabeza, desórdenes mentales, e incluso pude ocasionar la muerte, particularmente en los casos en los que no es detectada ni tratada a tiempo (2). A ello debemos sumar que la NCC ha sido considerada como

\footnotetext{
Laboratorio de Bioinformática y Biología molecular, Laboratorios de Investigación y Desarrollo, Facultad de Ciencias y Filosofía, Universidad Peruana Cayetano Heredia. Lima, Perú.

2 Laboratorio de Biotecnología y Biología molecular, Instituto Nacional de Salud (INS). Lima, Perú.

a Biólogo; ${ }^{\mathrm{b}}$ físico; ${ }^{\mathrm{c}} \mathrm{PhD}$ en control y prevención de enfermedades

Recibido: 11-04-13 Aprobado: 22-05-13
}

Citar como: León N, Padilla C, Pajuelo M, Sheen P, Zimic M. Cisteínoproteasas catepsinas L de Taenia solium: rol biológico en la infección y potencial uso para el inmunodiagnóstico de la neurocisticercosis. Rev Peru Med Exp Salud Publica. 2013;30(3):446-54. 
una causa importante de epilepsia sintomática en países en vías de desarrollo ${ }^{(1)}$.

\section{MORFOLOGÍA Y CICLO BIOLÓGICO DE Taenia solium}

En términos generales, T. solium es un gusano plano, cosmopolita, cuya anatomía se divide en escólex y estróbilo (cuerpo). El escólex presenta un conjunto de ganchos rostelares y cuatro ventosas, que permiten mantener estable al gusano en el intestino. El estróbilo está compuesto por segmentos unidos entre sí llamados proglótidos que nacen a nivel del cuello, y van madurando a lo largo del cuerpo, de tal manera que un proglótido maduro presenta órganos sexuales funcionales para la autofecundación, y el grávido contiene los huevos del gusano para su posterior liberación. Debido a que la tenia carece de un tracto digestivo, presenta en su superficie pequeñas extensiones o microtricas para la absorción de nutrientes y secreción de desechos. El huevo de la tenia contiene un embrión u oncósfera con seis ganchos, protegida principalmente por una estructura proteica llamada embrióforo. El cisticerco (metacéstodo) es una estructura translúcida que contiene a la larva de $T$. solium, esta se nutre y libera sus desechos a través de la superficie del cisticerco ${ }^{(3,4)}$.

El ciclo biológico de $T$. solium alterna entre el ser humano (huésped definitivo) causando teniosis, y el cerdo (huésped intermediario) causando cisticercosis. La tenia se desarrolla hasta el estadio adulto en el intestino delgado humano, donde se autofecunda (hermafrodita autosuficiente) y llega a producir miles de huevos. Estos alcanzan su madurez en los proglótidos grávidos, posteriormente son expulsados con las heces fecales. El cerdo es infectado al consumir alimentos contaminados con huevos, o proglótidos grávidos de la tenia adulta. Después que el cerdo ha ingerido los huevos, estos son estimulados por el ambiente ácido del estómago lo cual provoca que se liberen los embriones u oncósferas, estos luego atraviesan la pared intestinal y migran a diferentes partes del cuerpo por el torrente sanguíneo ${ }^{(5)}$. En el tejido muscular esquelético o nervioso del cerdo, las oncósferas suelen anclarse hasta convertirse en cisticercos, produciéndose la cisticercosis porcina. Cuando una persona consume carne infestada de cisticercos; las larvas se fijan en la pared interna del intestino delgado humano donde se desarrollan hasta gusanos adultos y nuevamente se repite el ciclo. Sin embargo, en algunas ocasiones, el hombre se convierte en el hospedero intermediario al consumir alimentos contaminados con huevos infectivos, pudiendo desarrollar cisticercosis en el músculo o en el sistema nervioso central ${ }^{(3,4)}$.
A pesar de que el Estado peruano ha sumado esfuerzos para desarrollar estrategias de salud pública para prevenir y enfrentar dos de las más importantes enfermedades zoonóticas parasitarias, aún no se ha logrado erradicar al helminto debido, entre otros motivos, a la complejidad del diagnóstico y a que se desconoce el mecanismo de infección del gusano.

\section{LA CISTICERCOSIS}

El primer caso descrito de cisticercosis humana en la duramadre de un individuo epiléptico fue a mediados del siglo XVI ${ }^{(6)}$. A pesar que ya había sido descrita la enfermedad, solo fue determinada como parasitaria cuando Malpighi en 1697 descubrió la naturaleza animal de los quistes y reportó la descripción del escólex. A finales del siglo XVIII se tenía suficiente información de la teniosis y la cisticercosis, más aún no se había dilucidado el ciclo biológico de $T$. solium, por lo que tampoco no se había conseguido relacionar la teniosis con la cisticercosis. Fue a inicios del siglo XIX cuando se describió la anatomía de la tenia, y se designó al cisticerco como Cysticercus cellulosae, nombre que quedó en desuso al demostrarse que el cisticerco era la forma larvaria de $T$. solium ${ }^{(4)}$. Van Beneden demostró que a partir de huevos infectivos podían desarrollarse los cisticercos en cerdos; sin embargo, se desconocía el curso de la infección. Kuchenmeister en 1852, relacionó la tenia con los cisticercos cuando alimentó a un condenado a la pena capital con cisticercos y reportó en su autopsia la presencia de tenia en el intestino, demostrando que la teniosis se desarrollaba a partir de la cisticercosis ${ }^{(6)}$. En concordancia con estas observaciones, Yoshino en 1933 reportó haber ingerido cisticercos y describió la expulsión de proglótidos durante dos años, convirtiéndose en su propio proveedor de huevos para diversos estudios ${ }^{(3)}$. RabielaCervantes et al. en 1982 reportaron que el cisticerco de la tenia puede presentarse de dos formas en el cerebro humano: la forma racemosa, caracterizada por una estructura de gran tamaño con una vesícula de múltiples lóbulos en forma de racimo de uvas; y la forma celulosa caracterizada por una vesícula pequeña ovalada y de fácil visualización del escólex ${ }^{(7)}$.

Entre los principales factores de riesgo asociados a la cisticercosis tenemos los inadecuados hábitos higiénicos, consumo de alimentos contaminados con huevos o infectados con cisticercosis (sobre todo de carne de cerdos enfermos), ausencia de saneamiento ambiental, carencia de tratamientos antiparasitarios y falta de campañas de educación comunitaria, concientización y capacitación de parte de las autoridades a la población ${ }^{(8,9)}$. Es por ello que 
para controlar y eliminar esta enfermedad, se han hecho esfuerzos a través de tratamientos antiparasitarios de poblaciones endémicas, desarrollando vacunas para cerdos y otras medidas que, lamentablemente, no han sido aplicadas satisfactoriamente en todos los países afectados.

\section{EPIDEMIOLOGÍA DE LA NEUROCISTICERCOSIS EN EL PERÚ}

Latinoamérica es una región endémica para la cisticercosis, se han informado casos de NCC en todos los países de la región, excepto en Chile, Argentina y Uruguay. En tanto que en México, Perú y Ecuador se ha señalado que, aproximadamente, la mitad de pacientes con crisis convulsivas de inicio tardío presentan evidencias de imágenes compatibles con NCC ${ }^{(10)}$. En 1999, luego de estudiar doce comunidades sudamericanas, Bern et al. informaron de 23512 a 39186 casos de NCC sintomáticos en Perú, y aproximadamente 400000 casos en Latinoamérica, encontraron además, una seroprevalencia de cisticercosis de 6 a 24\%, y una prevalencia en áreas de endemicidad de $6-10 \%$. Demostraron así que la NCC era endémica en las zonas montañosas y de la selva de Latinoamérica ${ }^{(11)}$.

Tradicionalmente, los estudios de cisticercosis en Perú han estado más orientados al diagnóstico y el tratamiento que a la epidemiología. Esta situación, sin embargo, ha ido cambiando en las últimas décadas, por lo que hoy en día se conoce mejor la epidemiología de la NCC en el Perú (12). Estudios seroepidemiológicos realizados entre 1990 y 1995 informaron seroprevalencias basales de cisticercosis entre 10 y $20 \%$ en la población general. Asimismo, la costa norte y la selva fueron identificadas como focos receptores de migración de zonas endémicas para NCC ${ }^{(12)}$. Los principales departamentos que presentan cisticercosis son: Tumbes, Apurímac, Junín, Cusco, Piura y San Martín, con prevalencias que oscilan entre 7 y $31 \%{ }^{(13,14)}$. En cambio la teniosis presentó una prevalencia entre 0,5 a $1 \%$ en pobladores que habitaban zonas endémicas ${ }^{(12)}$. A ello se suma que se ha reportado que solo en el manejo hospitalario el costo por paciente con NCC puede alcanzar 1100 dólares americanos ${ }^{(16,17)}$. La NCC es, además, la causa de al menos 50000 muertes anuales ${ }^{(18)}$. La variación de las prevalencias dependen principalmente de las medidas tomadas por las autoridades sanitarias en el manejo y destino de las heces humanas; de la relación entre humanos y cerdos; del control de la carne de cerdo parasitada; de las costumbres culturales en cuanto al consumo y manejo de la carne de cerdo, y los procedimientos para identificar y tratar a los portadores del parásito adulto ${ }^{(15,19)}$.
Asimismo, existen trabajos de investigación relacionados con la biología de $T$. solium, por ejemplo, aquellos vinculados con las diferentes clases de proteasas han permitido comprender mejor a este parásito, y ha ayudado no solo a esclarecer aspectos de su ciclo biológico y los diferentes roles que cumplen estas proteínas dentro de este ciclo, sino además han servido de sustrato para desarrollar potenciales usos para el inmunodiagnóstico de esta parasitosis.

\section{CLASIFICACIÓN DE LAS PROTEASAS: CISTEIINOPROTEASAS}

La Unión Internacional de Bioquímica ha reconocido cuatro clases de proteasas, según su mecanismo de acción: serinoproteasas, cisteínoproteasas, aspárticoproteasas y metaloproteasas. Dentro de estas existen seis familias que presentan residuos de aminoácidos funcionales arreglados en una configuración particular para formar un sitio activo, los miembros de cada familia han descendido de un ancestro común por evolución divergente (20). Las serinoproteasas incluyen dos familias: las serinoproteasas de mamíferos (quimiotripsina, tripsina, elastasa) y las serinoproteasas de bacterias (subtilisina), ambas familias difieren en la geometría del sitio activo y en el mecanismo enzimático. Por su parte, las metaloproteasas incluyen dos familias: la carboxipeptidasa pancreática de mamífero y la termolisina bacteriana, ambas dependen del zinc con sitios activos similares, pero con diferente estructura química. Por su parte, las aspárticoproteasas incluyen la penicilopepsina bacteriana, la pepsina de mamífero, la renina, la quimosina y las proteasas fúngicas. Asimismo, las cisteínoproteasas incluyen varias catepsinas lisosomales de mamíferos como las proteasas activadas por calcio citosólico (calpaínas), la papaína y la actidina que son proteasas de plantas. Las cisteínoproteasas tiene una gran similaridad con el grupo de las serinoproteasas, ya que las enzimas de ambas familias forman un enlace covalente intermedio ${ }^{(20)}$.

En mamíferos, las cisteínoproteasas han sido ampliamente estudiadas, y sus funciones están relacionadas con un conjunto de procesos intercelulares e intracelulares, como la invasión tumoral; la migración de células cancerosas; el procesamiento de antígenos y de prohormonas, la disgregación del tejido cartilaginoso en pacientes con artritis, reacciones alérgicas, resorción ósea, osteoporosis, y enfermedades neurológicas como el alzheimer (21). En el género Taenia los estudios de las catepsinas aún son escasos, y sus mecanismos de acción son poco conocidos. 


\section{CARACTERÍSTICAS GENERALES DE LA CATEPSINA L EN Taenia solium}

En estudios filogenéticos se observó una gran diversidad de catepsinas; y se atribuyó esta variación a los procesos de duplicación de genes y divergencia, las cuales habrían generado otras cisteínoproteasas con nuevas funciones. Por ejemplo, en los estadios de desarrollo de Fasciola hepatica se expresa y secreta catepsinas de tipo $L$ y $B$, mas no catepsina F. De hecho, más del $80 \%$ de proteínas secretadas por fasciolas adultas son cisteínoproteasas catepsinas $L^{(22)}$.

Entre las catepsinas $L$ recombinantes expresadas por $T$. solium se han descrito dos tipos. La primera es la cisteínoproteasa del metacéstodo de $T$. solium, la cual presenta características proteolíticas sobre la albúmina de suero bovino (ASB) y sobre el lgG humano (gracias a lo cual es capaz de evadir la respuesta inmune), mas no sobre el colágeno ${ }^{(23)}$. El segundo tipo de catepsinas es homóloga a la cisteínoproteasa catepsina $L$ de $T$. pisiformis, la cual es capaz de degradar IgG humano, ASB y fibronectina; que a diferencia de la anterior estas proteasas son secretadas por la larva y el estadio adulto ${ }^{(24)}$. En ambos casos se ha descrito motivos típicos ERFNIN, GNFD y GCNGG, y los aminoácidos conservados $\mathrm{Q}, \mathrm{C}, \mathrm{H}, \mathrm{N}$ y W, observados en la secuencia de aminoácidos de la cisteínoproteasa catepsina L. El motivo GNFD, tendría un rol importante en el procesamiento de la propapaína ${ }^{(25)}$, mientras que ERFNIN es muy conservado ${ }^{(26)}$. Otra característica importante de estas proteasas son las $\mathrm{N}$-glicosilaciones, las cuales son importantes para la actividad catalítica de estas enzimas ${ }^{(23,24)}$.

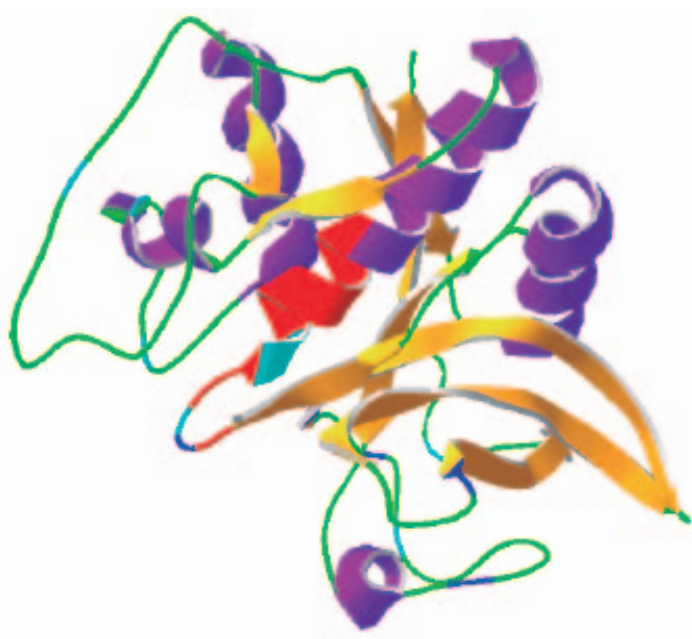

Figura 1. Estructura tridimensional de la proteína recombinante catepsina L del metacéstodo de Taenia solium predicha mediante un modelamiento por homología. Se muestra el dominio funcional conservado (rojo), las hojas beta plegadas (amarillo), las alfa hélices (morado), los loops de torsión (verde) y los puntos de coil-coiled (azul)
Otra estructura muy importante de las cisteínoproteasas catepsina L, son sus prorregiones (Figura 1), que cumplen roles cruciales para el adecuado foldeamiento de la proteína, además la prorregión de la catepsina $L$ ha sido implicada en el reconocimiento de la enzima hacia su sustrato, ya que presenta una región reconocida por las proteínas de membrana. La prorregión de la subfamilia catepsina $L$ está conformada de tres hélices $\alpha$ y una hoja $\beta$ corta, foldeado en un dominio discreto; donde la segunda hélice y la siguiente hoja $\beta$ forman una estructura en forma de horquilla ${ }^{(21)}$.

Debido a la importancia clínica e industrial de las cisteínoproteasas, se han estudiado muchos inhibidores, proponiéndose a las prorregiones como las mejores moléculas capaces de inhibir la actividad enzimática de la proteasa $^{(21)}$.

\section{ROL BIOLÓGICO DE LAS CATEPSINAS L DE PARÁSITOS}

Desde los primeros estudios de las interacciones entre parásitos y hospederos, se ha demostrado que los componentes de la matriz extracelular del hospedero, principalmente glucosaminoglicanos y glicoproteínas, tales como colágeno, elastina, fibronectina y laminina; desempeñan funciones primarias en la adherencia e invasión del parásito ${ }^{(27-30)}$. A pesar que no se conoce exactamente el mecanismo de infección de $T$. solium, se postula que las proteínas de superficie y de secreción de las oncósferas cumplirían funciones importantes para la invasión. Las glicoproteínas conocidas como lectinas son un grupo de moléculas que implicarían el reconocimiento celular o de adhesión de las oncósferas ${ }^{(31)}$. Además, se ha visto que la superficie de la larva o cisticerco de $T$. solium está muy glicosilada, por lo que se postula que cumple una función de adherencia ${ }^{(32,33)}$. La TSOL18, homóloga a EG95 de Echinococcus granulosus, es una proteína superficial de oncósfera que con su dominio fibronectina tipo III, puede adherirse a la matriz extracelular para favorecer su invasividad y puede activar la respuesta inmunológica del hospedero mediante sus epitopes conformacionales, haciendo de la TSOL18 un importante candidato de vacuna inmunoprotectiva contra la cisticercosis en cerdos ${ }^{(34-36)}$.

Al comparar la actividad peptidasa de productos secretorios y excretorios de la oncósfera de $T$. solium y T. saginata, con actividad serinopeptidasas (homólogas a quimiotripsina, tripsina, elastasa), cisteíno peptidasas (homólogas a catepsina L, catepsina B y calpaína) y aminopeptidasas (homóloga a peptidasa B), se han observado diferentes patrones de actividad catalítica. La actividad de enzimas similares a quimiotripsina 
fueron altas en ambos parásitos, siendo mayor en $T$. solium que en $T$. saginata. Del mismo modo, las que presentaban actividad de tripsina y catepsina B también fueron mayores en $T$. solium; mientras que las proteínas con actividad similar a catepsina $L$ y elastasa resultaron más altas en $T$. saginata. Las diferencias entre los perfiles de actividad de las peptidasas entre ambos parásitos, explicarían el rol modulador de las enzimas proteolíticas en la especificidad del hospedero para la invasión del cisticerco mediante la penetración de la mucosa intestinal, hipótesis que aún falta verificarse ${ }^{(5)}$.

Entre las proteínas de secreción tenemos a las cisteínoproteasas, que son importantes por su papel crítico en el ciclo de vida y patogenicidad de varios parásitos. Esta diversidad funcional está dada por su adaptación a diversos sustratos y su estabilidad en diferentes ambientes biológicos, además que estas proteasas cumplen roles claves en la inmunoinvasión parasitaria, en la activación de enzimas a nivel lisosomal, la invasión celular y de tejidos, en la eclosión, la muda, el enquistamiento y en el exquistamiento ${ }^{(37)}$.

La catepsina es una cisteínoproteasa capaz de degradar proteínas de la matriz extracelular, presenta residuos de cisteínas importantes para la actividad catalítica, y pertenece a la superfamilia papaína. Las catepsinas más estudiadas en los helmintos son la catepsina L, F, C y B. Se expresan en forma de zimógeno y requieren de la eliminación proteolítica de su prosegmento para su activación. Este proceso está afectado por la presencia de un lazo de oclusión que obstruye parcialmente la cavidad del sitio activo ${ }^{(38)}$ y restringe la especificidad del sustrato ${ }^{(39)}$. La prorregión de la subfamilia catepsina $L$ consiste de una pequeña hoja beta plegada y tres estructuras alfa-hélices, donde una alfa hélice forma un hairpin con la estructura paralela de la hoja beta plegada ${ }^{(21)}$.

Las catepsinas L, F y B se hallan en los perfiles de moléculas secretadas (secretoma o proteínas excretorias y secretorias), son sintetizadas por múltiples especies, desde bacterias hasta vertebrados superiores. En contraste, la catepsina C funciona como una aminopeptidasa de cadenas largas en la hidrólisis de macromoléculas ingeridas por el hospedero que han sido absorbidos dentro de la gastrodermis del lumen intestinal y no es encontrado en las secreciones de $F$. hepatica.

La expresión de las catepsinas en cada parásito es similar, pero su expresión dentro de cada parásito es considerablemente variable, por ejemplo, en $F$. hepatica la catepsina $L$ y $B$ son expresadas en estadios invasivos tempranos. El dominio $\mathrm{S} 2$ del sitio activo de estas cisteínas proteasas es específico para su sustrato. La especificidad del S2 está dada por residuos de aminoácidos que
Tabla 1. Rol biológico de las catepsinas $L$ y $B$ en organismos eucariotes, principalmente en los parásitos ${ }^{(47)}$

\begin{tabular}{|c|c|}
\hline Rol biológico & Parásito \\
\hline \multicolumn{2}{|c|}{$\begin{array}{l}\text { Factores de virulencia o } \\
\text { de interacción huésped- } \\
\text { parásito }\end{array}$} \\
\hline $\begin{array}{l}\text { Penetración } \\
\text { (dermis-tejido) }\end{array}$ & $\begin{array}{l}\text { Protozoarios (Trypanosoma, Leish- } \\
\text { mania, Tricomonas, Toxoplasma, } \\
\text { Plasmodium) }\end{array}$ \\
\hline Invasividad & $\begin{array}{l}\text { Platelmintos (Schistosoma, Fas- } \\
\text { ciola); Artrópodo (Dermatophagus) }\end{array}$ \\
\hline $\begin{array}{l}\text { Destrucción de las } \\
\text { proteínas del huésped }\end{array}$ & $\begin{array}{l}\text { Protozoarios (Tricomonas, Enta- } \\
\text { moeba, Plasmodium) }\end{array}$ \\
\hline $\begin{array}{l}\text { Degradación de la } \\
\text { matriz extracelular }\end{array}$ & $\begin{array}{l}\text { Platelmintos (Schistosoma, Para- } \\
\text { gonimus, Fasciola). }\end{array}$ \\
\hline $\begin{array}{l}\text { Evasión de la respuesta } \\
\text { inmune }\end{array}$ & $\begin{array}{l}\text { Protozoarios (Trypanosoma, Tri- } \\
\text { comonas, Giardia); platelmintos } \\
\text { (Schistosoma, Fasciola); nemáto- } \\
\text { dos (Haemonchus, Necator, Toxo- } \\
\text { cara); artrópodo (Dermatophagus) }\end{array}$ \\
\hline Nutrición & $\begin{array}{l}\text { Protozoarios (Entamoeba, Plasmo- } \\
\text { dium); nemátodos (Haemonchus, } \\
\text { Trichuris, Ancylostoma, Toxocara); } \\
\text { artrópodos (Dermatophagus) }\end{array}$ \\
\hline $\begin{array}{l}\text { Actividad } \\
\text { hemoglobinasa. }\end{array}$ & $\begin{array}{l}\text { Protozoarios (Tricomonas, Enta- } \\
\text { moeba, Plasmodium); platelmintos } \\
\text { (Schistosoma, Paragonimus, Fas- } \\
\text { ciola); nemátodos (Haemonchus, } \\
\text { Necator, Ascaris) }\end{array}$ \\
\hline \multicolumn{2}{|l|}{ Biología del parásito } \\
\hline $\begin{array}{l}\text { Crecimiento, desarrollo, } \\
\text { replicación }\end{array}$ & $\begin{array}{l}\text { Protozoarios (Trypanosoma, Leis- } \\
\text { hmania, Tricomonas); platelmintos } \\
\text { (Schistosoma, Paragonimus, Fas- } \\
\text { ciola); artrópodo (Dermatophagus); } \\
\text { nemátodos (Oncocerca, Brugia, } \\
\text { Ascaris) }\end{array}$ \\
\hline Enquistamiento & Protozoario (Giardia) \\
\hline
\end{tabular}

Adaptado de McKerrow et al. (47)

ocupan las posiciones $67,68,133,157,160$ y 205 dentro del sitio activo ${ }^{(22)}$. La catepsina L es muy importante para la penetración, infección y virulencia de varios parásitos hacia su hospedero ${ }^{(40-44)}$; pero además es capaz de degradar lgG del hospedero ${ }^{(45)}$ evadiendo de este modo la respuesta inmunológica ${ }^{(46,47)}$, otros roles biológicos de las catepsinas en parásitos se mencionan en la Tabla 1.

\section{CATEPSINA L EN EL INMUNODIAGNÓSTICO DE NEUROCISTICERCOSIS}

A pesar de que la cisticercosis humana es una enfermedad de gran importancia en la salud pública en Perú, la cual tiene además un componente económico-social, es necesario señalar que aún falta mejorar los medios de diagnóstico en zonas endémicas y las estrategias de saneamiento para la erradicación de esta enfermedad zoonótica.

La complejidad de la neurocisticercosis (NCC) radica en las variaciones en el número y localización de lesiones en cada individuo, además de las diferencias en la 
respuesta inmune del paciente frente al helminto. La NCC es una enfermedad pleomórfica, por lo que para su diagnóstico se consideran un conjunto de criterios clínicos y paraclínicos ${ }^{(48,49)}$. Los exámenes radiológicos, como la tomografía computarizada o resonancia magnética nuclear, son las pruebas más utilizadas para diagnosticar NCC en países desarrollados. A pesar de la efectividad de estas pruebas existen limitaciones durante la interpretación de los resultados, como el sobrelapamiento y localización aleatoria del cisticerco en el SNC, el reducido número de quistes o imágenes borrosas, lo cual resulta en algunos casos en hallazgos sorpresivos durante las intervenciones quirúrgicas terapéuticas. Otra limitación importante, sobre todo en países en vías de desarrollo, es que estas técnicas son muy costosas, por lo que su uso en las áreas endémicas de estos países es poco viable ${ }^{(49,50)}$, de allí la importancia de utilizar métodos inmunológicos para el diagnóstico.

Entre los ensayos inmunológicos utilizados para el diagnóstico de NCC tenemos las pruebas de hemaglutinación (IHA), radioinmunoensayo (RIA), ensayo inmunoabsorbente ligado a enzima (ELISA) y el Western blot con glicoproteínas ${ }^{(2)}$; esté último es altamente sensible y específico para el diagnóstico individual, pero su uso es poco práctico en sistemas de vigilancia epidemiológica ${ }^{(49)}$. Las muestras clínicas utilizadas en estos ensayos son los anticuerpos anticisticerco en sangre o líquido cefalorraquídeo (LCR) ${ }^{(49)}$; sin embargo, la detección específica de los anticuerpos no necesariamente indica una infección por cisticerco sino que también podría indicar una exposición anterior al parásito, existe además la posibilidad que se presenten reacciones cruzadas con epitopes antigénicos similares de otro parásito ${ }^{(51)}$. Además, se han dado casos de falsos positivos para NCC en pacientes con cisticercosis muscular o teniasis, y falsos negativos, en pacientes con quiste único o con NCC calcificado ${ }^{(9)}$. Es por eso que muchos grupos de investigación han estado trabajando, con diferentes antígenos más específicos de $T$. solium que puedan ser utilizados para el inmunodiagnóstico en campo.

Desde que Tsang et al., en 1989, caracterizaron glicoproteínas con pesos moleculares entre 10 a $50 \mathrm{kDa}$ con valor diagnóstico, se han estudiado las glicoproteínas del fluido quístico de $T$. solium con mayor interés ${ }^{(50)}$, los antígenos de excreción y secreción de oncósferas ${ }^{(52)}$, las proteínas sintéticas ${ }^{(53,54)}$ y proteínas con actividad similar a la catepsina $L^{(23,55)}$. Aún se siguen caracterizando otros antígenos que permitan hacer más rápido y eficiente las pruebas de diagnóstico para el adecuado tratamiento de la enfermedad.

Las cisteíno proteasas, debido a su papel fundamental durante el desarrollo y supervivencia del parásito, son candidatas importantes tanto para el desarrollo de pruebas de inmunodiagnóstico más eficientes, así como para el desarrollo de una vacuna(56-60). En 2005, Baig et al. purificaron y caracterizaron a partir de fluido de cisticerco, una cisteínoproteasa catepsina $L$ de $48 \mathrm{kDa}$, capaz de hidrolizar la lgG humana por lo que fue relacionada con la evasión del sistema inmune ${ }^{(46)}$. Posteriormente, en 2006 , Li et al. expresaron por primera vez una catepsina $L$ de $29 \mathrm{kDa}$ recombinante del metacéstodo de $T$. solium. Esta proteasa era capaz de degradar la albúmina de suero bovino (BSA) y el colágeno. Además, al ser evaluada para el inmunodiagnóstico mostró ser antigénica contra el suero de pacientes con cisticercosis, esparganosis o fasciolasis, con un pico débil o no antigénico contra paragonimiasis y clonorchiasis ${ }^{(23)}$.

En 2009, Zimic et al. purificaron a partir del fluido del cisticerco, dos fracciones de proteínas de $53 \mathrm{kDa}$ y $25 \mathrm{kDa}$, ambas con actividad similar a catepsina L, y estos antígenos se utilizaron para el diagnóstico humano de cisticercosis. El Western blot obtuvo una especificidad de $98 \%$ y una sensibilidad de $96 \%$ en pacientes con múltiples quistes, y una sensibilidad de $78 \%$ en pacientes con un solo quiste. Con respecto al ensayo inmunoabsorbente ligado a enzima (ELISA), presentó una especificidad del 92,7\%; mientras que la sensibilidad para pacientes con múltiples quistes fue de $98 \%$, y de $84 \%$ en pacientes con quiste único ${ }^{(55)}$.

Posteriormente, en 2011, Piña et al. propusieron un dot-ELISA altamente específico, sencillo, rápido y económico, que podría ser utilizado en regiones de bajos recursos económicos y en condiciones de campo. El dotELISA se desarrolló utilizando la fracción parcialmente purificada de proteínas con actividad catepsina $L$ de 53/25 kDa del cisticerco de T. solium ${ }^{(55)}$ para diagnosticar neurocisticercosis humana; y obtuvieron una especificidad de 99 a 100\%, con una leve reacción cruzada con Echinococcus granulosus (una sola muestra de los 18 sueros de pacientes con equinococcosis quística). Mientras que la sensibilidad varió según la ubicación de los quistes (94,4 a 100\% en pacientes con quistes extraparenquimales múltiples, 74,6 a $80,0 \%$ en pacientes con quistes parenquimales múltiples y 29,4 a $45,1 \%$ en individuos con un solo quiste parenquimal) ${ }^{(59)}$.

\section{CONCLUSIÓN}

La cisticercosis, especialmente la neurocisticercosis, es un problema de salud pública en nuestro país y en todo el mundo. A pesar de que el mecanismo de infección del cisticerco no es totalmente conocido, se sabe que existen muchas proteínas que trabajan concertadamente para hacer posible las interacciones parásito-hospedero. Las 
cisteínoproteinasas catepsina $\mathrm{L}$ cumplen un rol clave en las interacciones parásito-hospedero; así mismo, son buenos antígenos para el inmunodiagnóstico de cisticercosis, principalmente neurocisticercosis. Se han obtenido resultados prometedores que a futuro podrían facilitar un diagnóstico rápido a los pacientes de zonas endémicas y de bajos recursos económicos.
Investigación y desarrollo-LID) de la Universidad Peruana Cayetano Heredia, y al Dr. Pablo Ramírez de la Universidad Nacional Mayor de San Marcos.

Fuente de financiamiento: autofinanciado.

Conflicto de interés: los autores declaran no tener ningún conflicto de interés.

Agradecimientos: a los integrantes del Laboratorio de

Bioinformática y Biología molecular (Laboratorios de

\section{REFERENCIAS BIBLIOGRÁFICAS}

1. Ramírez-Zamora A, Alarcón T. Management of neurocysticercosis. Neurol Res. 2010;32(3):229-37. doi: $10.1179 / 016164110 \times 126442522605$ 92.

2. Deckers N, Dorny Pierre. Inmunodiagnosis of Taenia solium taeniosis/cysticercosis. Trends Parasitol. 2010;26(3):137-44. doi: 10.1016/j.pt.2009.12.008. Epub 2010 Jan 18.

3. Flisser A, Vargas-Parada L, Lacletter JP. Taenia solium: un parásito cosmopolita. Investigación y Ciencia. 2006:24-33.

4. Willms K, Vargas-Parada L, Laclette JP. Biología del parásito. En: Larralde C, De Aluja AS. Cisticercosis. Guía para profesionales de la salud. México, DF: FCE, Secretaría de Salud, Instituto Nacional de Salud Pública, Fundación Mexicana para la Salud; 2006. p. 19-40.

5. Zimic MJ, Infantes J, López C, Velásquez J, Farfán M, Pajuelo M, et al. Comparison of the peptidase activity in the oncosphere excretory/secretory products of Taenia solium and Taenia saginata. J Parasitol. 2007;93(4):72734.

6. Larralde C, De Aluja AS. Cisticercosis. Guía para profesionales de la salud. México, DF: FCE, Secretaría de Salud, Instituto Nacional de Salud Pública, Fundación Mexicana para la Salud; 2006.

7. Rabiela-Cervantes M, RivasHernandez A, RodriguezIbarra J, Castillo S, Cancino F. Anatomopathological aspects of human brain cysticercosis. En: Flisser A, Willms K, Laclette JP, Larralde C, Ridaura C, Beltran F. Cysticercosis: present state of knowledge and perspectives. Nueva York: Academic Press; 1982. p. 179-200.
8. Schantz PM. Taenia solium cysticercosis/teniosis is potentially erradicable disease: developing a strategy for actions and obstacles to overcome. En: García HH, Martínez SM, editors. Taenia solium: taeniasis / cysticercosis. Lima: Ed Universo; 1999. p. 215-7.

9. García HH, Gonzalez AE, Evans CA, Gilman RH; Cysticercosis Working Group in Peru. Taenia solium cysticercosis. Lancet. 2003;362(9383):547-56.

10. Capuñay C, Nilton S. Relación entre seropositividad a teniasis-cisticercosis por Taenia solium y neurocisticercosis asintomática en una zona endémica del Perú [Tesis para obtener el título de especialista en neurología]. Lima: Facultad de Medicina, Universidad Nacional Mayor de San Marcos; 2002.

11. Bern C, García HH, Evans C, Gonzales AE, Verástegui M, Tsang $\mathrm{VC}$, et al. Magnitude of the disease burden from neurocysticercosis in a developing country. Clin Infect Dis. 1999;29(5):1203-9.

12. Garcia HH, Gonzalez AE, Rodriguez $S$, Gonzalvez G, Llanos-Zavalaga F, Tsang VC, et al. Epidemiología y control de la cisticercosis en el Perú. Rev Peru Med Exp Salud Publica. 2010;27(4):592-7.

13. Cordero A, Miranda E, Segovia G, Cantoral V, Huarcaya I. Prevalencia de teniosis y seroprevalencia de cisticercosis humana en Pampa Cangallo, Ayacucho, Perú, 2008. Rev Peru Med Exp Salud Publica. 2010;27(4):562-8.

14. Escalante S. Cisticercosis I. Epidemiológica y clínica. Consideraciones anatómicas II. Cisticercosis porcina [Tesis Doctoral]. Lima: Facultad de Medicina, Universidad Peruana Cayetano Heredia; 1973.
15. Naquira C. Las zoonosis parasitarias: Problema de Salud Pública en el Perú. Rev Peru Med Exp Salud Publica. 2010;27(4):494-7.

16. Ferrer E. Teniosis/Cisticercosis: del diagnóstico convencional al diagnóstico molecular. Salus. 2007;11(Supl 1): 5761.

17. García HH, Gonzales A, Martínez SM, Gilman RH. Teniasis/cisticercosis por Taenia solium, un serio problema de salud pública en el Perú. Lima: Ministerio de Salud del Perú; 2001.

18. Gemmell M, Matyas Z, Pawloski Z, Soulsby EJL, Larralde C, Nelson GS. Guidelines for surveillance, prevention and control of taeniosis/cisticercosis. Geneva: World Health Organization WHO/VPH/83.49; 1983.

19. Garcia HH, Gonzalez AE, Gilman $\mathrm{RH}$; Cysticerosis Working Group in Peru. Diagnosis, treatment and control of Taenia solium Cysticercosis. Curr Opin Infect Dis. 2003;16(5):411-9.

20. Beynon RJ, Bond JS. Proteolytic enzymes: a practical approach. New Cork: Press at Oxford University Press; 1994.

21. Groves MR, Coulombe R, Jenkins J, Cygler M. Structural basis for specificity of papain-like cysteine protease proregions toward their cognate enzymes. Proteins. 1998;32(4):504-14.

22. Stack C, Dalton JP, Robinson MW. The phylogeny, estructure and function of trematode cysteine proteases, with particular emphasis on the Fasciola hepatica cathepsin L family. En: Robinson MW, Dalton J. Cysteine proteases of pathogenic organisms. Springer: Landes Bioscience and Springer Science+Business Media; 2011. 
23. Li AH, Moon SU, Park YK, Na BK, Hwang MG, Oh CM, et al. Identification and characterization of a cathepsin L-like cysteine protease from Taenia solium metacestode. Vet Parasitol. 2006;141(3-4):251-9.

24. Wang Q, Zhang S, Luo X, Hou J, Zhu $\mathrm{X}$, Cai $\mathrm{X}$. Cloning and characterization of a cathepsin L-like cysteine protease from Taenia pisiformis. Vet Parasitol. 2013 1;194(1):26-34. doi: 10.1016/j. vetpar.2012.12.055.

25. Vernet T, Berti PJ, de Montigny C, Musil R, Tessier DC, Ménard R, et al. Processing of the papain precursor. The ionization state of a conserved amino acid motif within the Pro region participates in the regulation of intramolecular processing. J Biol Chem. 1995;270(18):10838-46.

26. Karrer KM, Peiffer SL, DiTomas ME. Two distinct gene subfamilies within the family of cysteine protease genes. Proc Natl Acad Sci U S A. 1993;90(7):3063-7.

27. Furtado GC, Cao Y, Joiner KA. Laminin on Toxoplasma gondii mediates parasite binding to the beta 1 integrin receptor alpha 6 beta 1 on human foreskin fibroblasts and Chinese hamster ovary cells. Infect Immun. 1992;60(11):4925-31.

28. Hayman JR, Southern TR, Nash TE. Role of sulfated glycans in adherence of the microsporidian Encephalitozoon intestinalis to host cells in vitro. Infect Immun. 2005;73(2):841-8.

29. Atzingen MV, Barbosa AS, De Brito T, Vasconcellos SA, de Morais ZM, Lima DM, et al. Lsa21, a novel leptospiral protein binding adhesive matrix molecules and present during human infection. BMC Microbiol. 2008;8:70. doi: 10.1186/1471-21808-70.

30. Kulkarni MM, Jones EA, McMaster WR, McGwire BS. Fibronectin binding and proteolytic degradation by Leishmania and effects on macrophage activation. Infect Immun. 2008;6(4):1738-47. doi: 10.1128/ IAI.01274-07.

31. Lis $\mathrm{H}$, Sharon N. Lectins: Carbohydrate-Specific Proteins That Mediate Cellular Recognition. Chem Rev. 1998;98(2):637-74.

32. Landa A, Merchant MT, Willms K, Laclette JP. Purification and ultrastructural localization of surface glycoproteins of Taenia solium (Cestoda) cysticerci. Int J Parasitol. 1994;24(2): 265-9.
33. Restrepo BI, Obregón-Henao A, Mesa M, Gil DL, Ortiz BL, Mejía JS, et al. Characterisation of the carbohydrate components of Taenia solium metacestode glycoprotein antigens. Int J Parasitol. 2000;30(6):689-96.

34. Lightowlers MW, Flisser A, Gauci CG, Heath DD, Jensen O, Rolfe $R$. vaccination against cysticercosis and hydatid disease. Parasitol Today. 2000;16(5):191-6.

35. Assana E, Gauci CG, Kyngdon CT, Zoli AP, Dorny P, Geerts S, et al. Antibody responses to the host-protective Taenia solium oncosphere protein TSOL18 in pigs are directed against conformational epitopes. Parasite Immunol. 2010;32(6):399-405.

36. Zimic M, Gutiérrez AH, Gilman RH, López C, Quiliano M, Evangelista W, et al. Inmunoinformatics prediction of linear epitopes from Taenia solium TSOL18. Bioinformation. 2011;6(7): 271-4.

37. Sajid M, McKerrow JH. Cystein protease of parasitic organism. Mol Biochem Parasitol. 2002;120(1):1-21.

38. Musil D, Zucic D, Turk D, Engh RA, Mayr I, Huber R, et al. The refined 2.15 $\mathrm{A}^{\circ} \mathrm{X}$-ray crystal structure of human liver cathepsin B: the structural basis for its specificity. EMBO J. 1991;10(9):232130.

39. Quraishi O, Nägler DK, Fox T, Sivaraman J, Cygler M, Mort JS, et al. The occluding loop in cathepsin $\mathrm{B}$ defines the $\mathrm{pH}$ dependence of inhibition by its propeptide. Biochemistry. 1999;38(16):5017-23.

40. Harmsen MM, Cornelissen JB, Buijs HE, Boersma WJ, Jeurissen SH, van Milligen FJ. Identification of a novel Fasciola hepatica cathepsin L protease containing protective epitopes within the propeptide. Int J Parasitol. 2004;34(6):675-82.

41. Tantrawatpan C, Maleewong W, Wongkham C, Wongkham S, Intapan PM, Nakashima K. Serodiagnosis of human fascioliasis by a cystatin capture enzyme-linked immunosorbent assay with recombinant Fasciola gigantica cathepsin L antigen. Am J Trop Med Hyg. 2005;72(1):82-6.

42. Cornelissen JB, Gaasenbeek CP, Boersma W, Borgsteede FH, van Milligen FJ. Use of a pre-selected epitope of cathepsin-L1 in a highly specific peptide-based immunoassay for the diagnosis of Fasciola hepatica infections in cattle. Int $\mathrm{J}$ Parasitol. 1999;29(5):685-96.
43. Cordova M, Herrera P, Nopo L, Bellatin J, Naquira C, Guerra H, et al. Fasciola hepatica cysteine proteinases: immunodominant antigens in human fascioliasis. Am J Trop Med Hyg. 1997;57(6):660-6.

44. Córdova M, Reátegui L, Espinoza JR. Immunodiagnosis of human fascioliasis with Fasciola hepatica cysteine proteinases. Trans R Soc Trop Med Hyg. 1999;93(1):54-7.

45. Baig S, Damian RT, Morales-Montor J, Olecki P, Talhouk J, Hashmey R, et al. Characterization of excretory/ secretory endopeptidase and metalloaminopeptidases from Taenia crassiceps metacestodes. J Parasitol. 2005;91(5):983-7.

46. Baig S, Damian RT, Molinari JL, Tato $\mathrm{P}$, Morales-Montor J, Welch $\mathrm{M}$, et al. Purification and characterization of a metacestode cysteine proteinase from Taenia solium involved in the breakdown of human IgG. Parasitology. 2005;131(Pt 3):411-6.

47. McKerrow JH, Caffrey C, Kelly B, Loke P, Sajid M. Proteases in parasitic diseases. Annu. Rev Pathol. 2006;1:497-536.

48. Kalra V, Sethi A. Chilhood neurocysticercosis--epidemiology, diagnosis and course. Acta Paediatr Jpn. 1992;34(3):365-70.

49. Del Brutto OH. Neurocisticercosis: actualización en diagnóstico y tratamiento. Neurologia. 2005;20(8):412-8.

50. Otake M, Sako Y, Nakao M, Yamasaki $\mathrm{H}$, Nkaya K, Ito A. Evaluation of purified Taenia solium glycoproteins and recombinant antigens in the serologic detection of human and swine cysticercosis. J Infec Dis. 2006;194(12):1783-90.

51. Garcia HH, Gonzalez AE, Gilman RH, Palacios LG, Jimenez I, Rodriguez $S$, et al. Short report: transient antibody response in Taenia solium infection in field conditions-a major contributor to high seroprevalence. Am J Trop Med Hyg. 2001;65(1):31-2.

52. Verastegui M, Gilman RH, Garcia $\mathrm{HH}$, Gonzales AE, Arana Y, Jeri C, et al. Prevalence of antibodies to unique Taenia solium oncosphere antigens in taeniasis and human and porcine cisticercosis. Am J Trop Med Hyg. 2003;69(4):438-44.

53. Scheel CM, Khan A, Hancock K, García HH, Gonzales AE, Gilman $\mathrm{RH}$, et al. Serodiagnosisticercosis using synthetic 8-kd proteins: comparison 
of assay formats. Am J Trop Med Hyg. 2005;73(4):771-6.

54. Tsang VC, Brand JA, Boyer AE. An enzyme-linked immunoelectrotransfer blot assay and glycoprotein antigens for diagnosis human cysticercosis (Taenia solium). J Infect Dis. 1989;159(1):50-9.

55. Zimic M, Pajuelo M, Rueda D, López C, Arana Y, Castillo Y, et al. Utility of a protein fraction with cathepsin L-like activity purified from cysticercus fluid of Taenia solium in the diagnosis of human cysticercosis. J Trop Med Hyg. 2009;80(6):964-70.

56. Sako Y, Yamashi H, Nakaya K, Nakao $\mathrm{M}$, Ito A. Cloning and characterization of cathepsin L-like peptidases of Echinococcus multilocularis metacestodes. Mol Biochem Parasitol. 2007;154(2):181-9.
57. Cortez AP, Rodrigues AC, García HA, Neves L, Batista JS, Bengaly Z, et al. Cathepsin L-like genes of Trypanosoma vivax from Africa and South Americacharacterization, relationships and diagnostic implications. Mol Cell Probes. 2009;23(1):44-51.

58. Malagón D, Díaz-López M, Benítez R, Adroher FJ. Cathepsin B-and L-like cysteine protease activities during the in vitro development of Hysterothylacium aduncum (Nematoda: Anisakidae), a worldwide fish parasite. Parasitol Int. 2010;59(1):89-92.

59. Piña R, Gutierrez AH, Gilman RH, Rueda D, Sifuentes C, Flores $\mathrm{M}$, et al. A dot-ELISA using a partially purified cathepsin L-like protein fraction from Taenia solium cysticerci, for the diagnosis of human neurocysticercosis. Ann Trop Med Parasitol. 2011;105(4):311-8.

60. Zimic M, Pajuelo M, Gilman RH, Gutiérrez AH, Rueda D, Flores M, et al. The highly antigenic $53 / 25 \mathrm{kDa}$ Taenia solium protein fraction with cathepsin L like activity is present in the oncosphere/cysticercus and induces non-protective $\operatorname{IgG}$ antibodies in pigs. Vet Immunol Immunopathol. 2012;145(1-2):171-8.

Correspondencia: Nancy León Janampa. Dirección: Universidad Peruana Cayetano Heredia, Av. Honorio Delgado, Lima-Perú. Teléfono: (51) 3190000 anexo 2406 Correo:naleja_2386@hotmail.com

\section{Consulte las ediciones anteriores de la} Revista Peruana de Medicina Experimental y Salud Pública en

\section{www.scielosp.org}

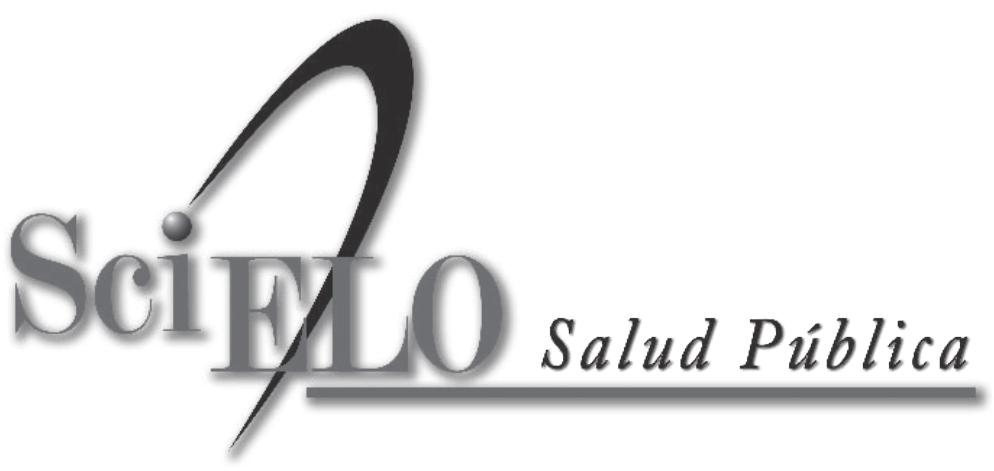

